\title{
Hernia perineal en el perro, un estudio de prevalencia de 81 casos
}

\author{
Perineal hernia in the dog, a prevalence study of 81 cases \\ A Ramírez ${ }^{\mathrm{a}}$, N Pastor ${ }^{\mathrm{a}}$, ME Durán ${ }^{\mathrm{b}}$, A Gutiérrez $^{\mathrm{a}}$, LJ Ezquerra $^{\mathrm{b}}$ \\ apepartamento de Cirugía, Hospital Clínico Veterinario, Facultad de Veterinaria, \\ Universidad de Extremadura, Cáceres, España. \\ ${ }^{\text {b}}$ Departamento de Medicina Animal, Hospital Clínico Veterinario, Facultad de Veterinaria, \\ Universidad de Extremadura, Cáceres, España.
}

\begin{abstract}
SUMMARY
The aim of this is study was to analyse perineal hernia's prevalence in the population, describe its characteristics and to evaluate different surgical techniques used and their results. The study design is a retrospective study of perineal hernia's cases in the Veterinary Teaching Hospital of the University of Extremadura. Perineal hernia cases are $0.96 \%$ of the population. Males and females represent $98.2 \%$ and $1.2 \%$, respectively. Our population with perineal hernia has an average age of 8,69 years, and a standard deviation of 2.19 years. The average weight body was $16.4 \mathrm{~kg} \pm 11.44 \mathrm{~kg}$. We found 18 bilateral perineal hernias, 40 right unilateral hernias and 20 left unilateral; the total number was 96 perineal hernias. Sixty seven patients underwent surgery, and a total of 80 perineal hernias were repaired. The most frequent clinical signs were perineal bulge and defecation difficulty. Bladder retroflexion appeared in $13.6 \%$ of the cases and the prostate was affected in the $21 \%$. It was noted that bilateral perineal hernia patients had increased susceptibility to bladder retroflexion. The techniques used were as follows: anatomical or conventional (7.55\%), transposition of the internal obturator muscle (67.92\%), anatomical with polypropylene mesh (11.32\%), transposition of the internal obturator muscle with polypropylene mesh (13.22\%). The transposition of the internal obturator muscle obtained a recurrence's rate of $2.8 \%$. Complications's rate and recurrence's rate on perineal herniorraphy were $9.84 \%$ and $14.8 \%$, respectively.
\end{abstract}

Key words: perineal hernia, dog, surgery.

\section{RESUMEN}

Este estudio se llevó a cabo con el fin de analizar la prevalencia de la hernia perineal en la población, describir las características de la misma y evaluar las distintas técnicas empleadas para la herniorrafia y sus resultados. Se realizó un estudio retrospectivo de los casos de hernia perineal en el Hospital Clínico Veterinario de la Universidad de Extremadura. Los casos de hernia perineal representan 0,96\% de la población. Los machos constituyen un $98,2 \%$ y las hembras $1,2 \%$. Los pacientes tienen una edad media de 8,69 años, y una desviación típica de 2,19 años. El peso fue de $16,4 \mathrm{~kg} \pm 11,44$ $\mathrm{kg}$ (media \pm desviación típica). Se encontraron 18 hernias perineales bilaterales, 40 unilaterales derechas y 20 unilaterales izquierdas, con 96 hernias perineales. Fueron intervenidos 67 pacientes, sumando 80 hernias perineales operadas. Presentaron retroflexión de vejiga el 13,6\% de los casos y afectación de la próstata el $21 \%$. Se observó que los pacientes con hernia perineal bilateral tenían mayor predisposición a tener la vejiga en retroflexión. Las técnicas empleadas fueron las siguientes: anatómica o convencional (7,55\%), transposición del obturador interno (67,92\%), anatómica con malla $(11,32 \%)$, transposición del obturador interno con malla $(13,22 \%)$. La técnica de transposición del obturador interno obtuvo 2,8\% de recidivas. La tasa de complicaciones y de recidiva tras la herniorrafia perineal se eleva al $9,84 \%$ y $14,8 \%$, respectivamente.

Palabras clave: hernia perineal, perro, cirugía.

\section{INTRODUCCIÓN}

Las hernias perineales ocurren como consecuencia del deterioro de la función del diafragma pélvico (Hosgood 1995, Fossum 2007, Pérez-Gutiérrez y col 2011). El diafragma pélvico está formado por los músculos coccígeo, elevador del ano, obturador interno, glúteo superficial y esfínter anal (Bellenger y Canfield 2003). Las hernias perineales pueden ser unilaterales o bilaterales. Aparecen más frecuentemente lateral al ano, pero también pueden aparecer ventral a este (Tobias 2010).

Aceptado: 07.07.2014.

* Avda. de la Universidad s/n 10003, Cáceres, España; anazerimarp@gmail.com
La etiología de esta enfermedad está relacionada con la presencia de hormonas masculinas, la edad, la raza y la debilidad muscular congénita o adquirida (Bellenger y Canfield 2003, Fossum 2007). Afectan más frecuentemente a machos, la patología en las hembras suele ser de origen traumático o asociadas a la preñez (Sontas y col 2008), y aparecen menos comúnmente. Los pacientes deberán ser castrados, para reducir la influencia hormonal sobre la pared perineal (Hayes y col 1978). La castración deberá ser previa a la herniorrafia.

Una vez que comienza el deterioro del diafragma pélvico, que empieza a perder su función de soporte, la grasa peritoneal se coloca entre los músculos. Esta grasa ocupará la fisura del músculo elevador del ano, por la que pasa el nervio obturador, e interrumpirá total 
o parcialmente las fibras de dicho nervio y da lugar a una atrofia neurogénica de los músculos inervados por él (Bellenger y Canfield 2003).

Debido a la atrofia del músculo elevador del ano, el contenido abdominal puede pasar al saco herniario. Este contenido suele ser intestino y tejido adiposo abdominal. La vejiga y la próstata pueden aparecer también en la hernia (Head y Fracis 2002, Pratummintra 2013).

El diagnóstico diferencial de la tumoración perineal comprende: hernia perineal, neoplasia perineal, hiperplasia de glándula perianal, saculitis anal, atresia anal, neoplasia del saco anal y tumor vaginal (Fossum 2007).

En la resolución de la hernia perineal se han descrito varias técnicas: herniorrafia simple o anatómica, transposición del músculo obturador interno, transposición del músculo glúteo superficial, y el uso de implantes. Estas técnicas pueden usarse solas o combinadas. Como técnicas complementarias a estas se pueden usar colopexia, deferentopexia y cistopexia (Bellenger y Canfield 2003).

El objetivo de este estudio es analizar la prevalencia de la hernia perineal en el Hospital Clínico Veterinario de la Universidad de Extremadura, describir las características de la misma y evaluar las distintas técnicas empleadas para la herniorrafia, así como sus resultados.

\section{MATERIAL Y MÉTODOS}

Se realizó un estudio clínico retrospectivo de los casos atendidos en la consulta de Cirugía de pequeños animales del Hospital Clínico Veterinario de la Universidad de Extremadura, desde octubre de 1986 hasta julio de 2011. En este estudio se analizaron los datos hallados en las historias clínicas.

Para la herniorrafia, los pacientes deben colocarse en decúbito ventral, con el rabo fijado hacia delante y la cadera elevada. La zona quirúrgica a abordar se rasura previamente y se hace una preparación aséptica de la zona. También se procede a vaciar el recto y se coloca una sutura en bolsa de tabaco en el ano, para impedir la salida de heces durante la cirugía. Para resolver la hernia perineal fueron empleadas distintas técnicas como la anatómica (Fossum 2007), la transposición del obturador interno (Fossum 2007), la anatómica con malla de polipropileno y la transposición del obturador interno con malla de polipropileno. La deferentopexia (Fossum 2007), la colopexia (Bellenger y Canfield 2003) y la cistopexia (Bellenger y Canfield 2003) se emplearon como técnicas complementarias para mejorar el resultado de la herniorrafia.

El análisis de los datos se ha realizado con el programa estadístico SPSS, versión 19 para Windows. Se ha hecho una estadística descriptiva y para saber si las diferencias son estadísticamente significativas se ha utilizado la Prueba Exacta de Fisher y la Prueba Chi-Cuadrado de Pearson. Se ha considerado estadísticamente significativo un P-valor de 0,05 o inferior.

\section{RESULTADOS}

Se ha encontrado una prevalencia del $0,96 \%$ de hernia perineal, contando 81 casos entre 8.439 casos quirúrgicos en el Hospital. El 98,8\% son machos y el $1,2 \%$ son hembras, entre los machos había 2 criptórquidos abdominales, que representan $2,47 \%$ de los casos con hernia perineal.

La edad de los perros con hernia perineal oscila entre los 3 y los 14 años, con una media de 8,69 años y una desviación típica de 2,19 años, la mediana es 9 años y la moda 8 años. El 75\% de los animales tienen entre 6 y 11 años. La raza de los pacientes con hernia perineal es muy heterogénea; las razas Pequinés, Pastor Alemán y Yorkshire son las que obtuvieron mayor frecuencia, dentro de las razas puras.

Con la información recogida en las historias clínicas, se encontraron 18 hernias perineales bilaterales, 40 hernias unilaterales derechas y 20 unilaterales izquierdas. Contabilizando las hernias bilaterales como 2 hernias, se obtienen 96 hernias perineales estudiadas. Entre todos los casos hay tres de ellos que no se sabe a qué tipo de hernia correspondían, considerándose los mismos como casos perdidos en la estadística. De todos estos casos, en 67 de ellos el propietario respectivo aceptó el tratamiento quirúrgico, con 80 hernias perineales operadas. La prueba estadística revela que existen evidencias estadísticamente significativas en el tipo de hernia, siendo la hernia perineal derecha la más destacada.

Los pacientes que acudieron a la consulta llevaban con la enfermedad entre 9 días y 60 meses, la media fue de 5,75 meses y la desviación típica de 8,84 meses. Los signos clínicos más frecuentes fueron abultamiento perineal $(77,8 \%)$, dificultad para defecar $(54,3 \%)$, dificultad para orinar $(6,2 \%)$, obstrucción urinaria $(3 \%)$, y en menor medida mal estado general, deshidratación o vómitos. En el 55,6\% de los casos se realizó radiografías simples o de contraste vesical.

En 11 casos apareció la vejiga en retroflexión, representando $13,6 \%$ del total de la población. Se obtuvieron evidencias estadísticamente significativas entre el tipo de hernia y la probabilidad de aparecer la vejiga herniada $(\mathrm{P}=0,006)$, correspondiendo el $63,6 \%$ de los casos de vejiga herniada a hernias perineales bilaterales (figura 1). Por tanto existe mayor probabilidad de encontrarse la vejiga herniada si hay una hernia perineal bilateral.

En el 13,6\% de las hernias perineales se observó un aumento del tamaño de la próstata y esta estaba incluida en la hernia en el 7,4\% de los casos. Los casos en los que no se realizó la castración representan 17,9\%, los castrados previamente a la cirugía son $3,6 \%$ y los castrados en el momento en que se realiza la cirugía reparadora de la hernia constituyen $78,6 \%$. No se encontraron diferencias significativas en cuanto a la aparición de recidivas relacionadas con la castración o no, pero son pocos los animales que no se han castrado. 


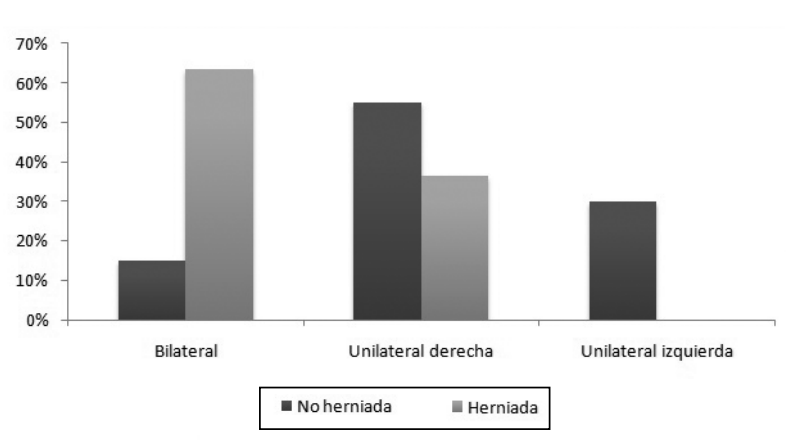

Figura 1. Estado de la vejiga clasificada en función del tipo de hernia.

Bladder condition by type of hernia.

El 65,3\% de los casos recibieron tratamiento quirúrgico, el $3,7 \%$ recibieron tratamiento conservador y al $31 \%$ no se le administró ningún tratamiento, por decisión de su propietario. Las técnicas empleadas para resolver la hernia fueron las siguientes: la técnica anatómica que se empleó en $7,55 \%$ de los casos, la técnica de transposición del músculo obturador interno en un $67,92 \%$, la técnica anatómica con malla de polipropileno que se usó en 11,32\% y la técnica de transposición del obturador interno con malla que se combinó en $13,21 \%$.

Las técnicas complementarias a la herniorrafia se utilizaron en el 47,8\%, realizándose en el 31,5\% deferentopexias, $14,8 \%$ colopexias y en $1,5 \%$ cistopexia. La deferentopexia y la colopexia se combinaron en el 11,1\% de los casos.

Las recidivas constituyeron $13,32 \%$ del total de los casos intervenidos. El porcentaje de recidivas obtenido para cada técnica es el siguiente: anatómica, 50\%; transposición obturador interno, 2,8\%; anatómica con malla, 33,3\%; transposición del obturador interno con malla, 14,3\% (cuadro 1). Hemos obtenido evidencias estadísticamente significativas $(\mathrm{P}=0,009)$, siendo la transposición del obturador interno la técnica más fiable para la herniorrafia perineal.

Las complicaciones surgidas de los casos quirúrgicos, sin tener en cuenta las recidivas, representan el $8,2 \%$ en nuestra población (figura 2). Aparecieron las siguientes: infección de la incisión $(1,64 \%)$, laxitud de la piel $(1,64 \%)$, seroma $(3,28 \%)$ y no resolución de la hernia $(1,64 \%)$.

\section{DISCUSIÓN}

La hernia perineal es la consecuencia del debilitamiento de los músculos del diafragma pélvico, que no logran sostener la pared rectal. Esto ocasiona distensión rectal y su consecuente deterioro en la defecación (Fossum 2007). En nuestro hospital se presentó en el 0,96\% de los casos quirúrgicos. Las características de nuestra población con hernias perineales se asemejan a las descritas en la bibliografía, afectando en $98,2 \%$ a machos. La presencia de hembras no suele ser frecuente, se han descrito casos debidos a traumatismos, asociadas a la preñez (Sontas y col 2008), y un caso de pseudohermafroditismo (Szabo 2008). La predisposición a la aparición de la hernia se debe a la presencia de hormonas masculinas. Recientemente se ha descrito el papel de la hormona caspasa-3 activada en esta patología, y se ha demostrado que los pacientes con hernia perineal presentan un mayor número de receptores frente a la hormona sobre el músculo elevador del ano (Pérez-Gutiérrez y col 2011).

Entre los machos el 2,47\% eran criptórquidos. Ya se había descrito anteriormente la aparición de criptorquidia asociada a las hernias perineales (Hosgood 1995), sin embargo, no hemos podido demostrar estadísticamente que exista una relación entre la criptorquidia y la aparición de hernias perineales. La edad media de aparición de esta patología en nuestro caso es 8,69 $\pm 2,19$ años, el 75\% de los animales tenían entre 6 y 11 años. La hernia perineal es una enfermedad degenerativa del diafragma pélvico, por tanto, lo habitual es que aparezca en animales geriátricos. Otros autores describen una edad media de aparición de 9,4 años (Hosgood 1995), 9,8 años (Bellenger y Canfield 2003), 9,7 años (Brissot y col 2004).

No se ha encontrado una predisposición racial para padecer esta afección, aunque las razas puras más representadas en nuestro estudio fueron el Pequinés y el Pastor Alemán. En otros estudios revisados (Hosgood 1995, Brissot y col 2004, Szabo 2007) tampoco se ha observado predisposición racial, sin embargo hay autores que aseguran que la raza Pequinés prodría ser de las más afectadas por esta enfermedad (Weaber y Omamegbe 1981, White y Herrtage 1981).

Cuadro 1. Tasa de recidiva en función de la técnica quirúrgica. Recurrence rates based on the surgical technique.

\begin{tabular}{llcccc}
\hline & & & \multicolumn{2}{c}{ Técnica } \\
\cline { 3 - 6 } & & Anatómica & $\begin{array}{c}\text { Transposición } \\
\text { obturador } \\
\text { interno }\end{array}$ & $\begin{array}{c}\text { Anatómica }+ \\
\text { malla }\end{array}$ & $\begin{array}{c}\text { Transposición } \\
\text { obturador } \\
\text { interno + malla }\end{array}$ \\
\hline Recidivas & Sí & $50,0 \%$ & $2,8 \% *$ & $33,3 \%$ & $14,3 \%$ \\
& No & $50,0 \%$ & $97,2 \%$ & $66,7 \%$ & $85,7 \%$ \\
\hline
\end{tabular}

* Estadísticamente significativo $\mathrm{P}<0,05$. 


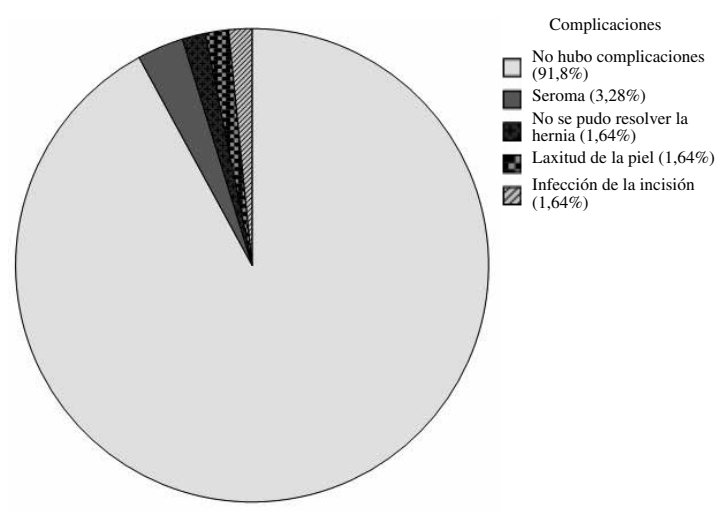

Figura 2. Gráfico de sectores en el que podemos ver detalladamente los porcentajes de las complicaciones.

Pie chart showing in detail the complications rates.

La hernia perineal suele aparecer con más frecuencia de manera unilateral y en el lado derecho (Hardie y col 1983, Mann 1993, Hosgood 1995), como ocurre en nuestra población, en la que el $51,28 \%$ son hernias perineales unilaterales derechas. Se ha considerado que puede ser debido a una debilidad intrínseca del lado derecho del periné por una deficiencia de peso de los músculos del lado derecho frente a los del lado izquierdo del cuerpo (Canfield 1986). La duración media de los signos clínicos es muy variable en las referencias encontradas y los signos clínicos de abultamiento perineal y dificultad para defecar parecen ser los más frecuentes (Hosgood 1995, Szabo 2007).

El 13,6\% de los pacientes tenían la vejiga contenida en la hernia, se han descrito porcentajes del 18\% (Burrows y Harvey 1973), 20\% (Hosgood 1995) y hasta 29\% (Brissot y col 2004). Debe considerarse la posición de la vejiga cuando se nos presenta una hernia perineal. Nuestros datos estadísticos revelan que existe mayor probabilidad de encontrar la vejiga herniada si se diagnostica una hernia perineal bilateral $(\mathrm{P}=0,006)$, hecho que no se había publicado previamente.

El 21\% de pacientes tenía afectación prostática, las hernias perineales suelen estar asociadas con patologías de la próstata. Podemos encontrarla contenida en la hernia, hiperplasia prostática o quistes prostáticos (Brissot y col 2004, Head y Francis 1978, Venuk y col 2008), en cualquier caso es un factor a tener en cuenta al evaluar y tratar una hernia perineal. El 82,2\% de los pacientes fueron castrados previamente o durante la cirugía; el riesgo de recidiva para los animales no castrados aumenta en 2,7 veces (Hayes y col 1978), aunque en nuestro estudio no había diferencias estadísticamente significativas porque son pocos los animales que no se han castrado.

Entre los 81 casos de hernia perineal recibieron tratamiento quirúrgico el $65,3 \%$, de estos en el $67,92 \%$ se utilizó la técnica de transposición del obturador interno. La transposición del obturador interno es la que menor tasa de recidivas ha obtenido; con nuestros resultados puede afirmarse que es la técnica más segura y fiable para la herniorrafia perineal. La tasa de recidiva de la enfermedad depende de diversos factores. Hay autores que señalan que la inexperiencia del cirujano podría ser un factor clave, con unas tasas de recidiva muchísimo mayores para cirujanos inexpertos comparado con expertos, $70 \%$ (Burrows y Harvey 1973) y 10\% (Matteison 1989), respectivamente. La técnica quirúrgica también puede considerarse un factor clave en la tasa de recidiva, siendo muy variable dependiendo de la técnica empleada. Para la transposición del músculo obturador interno hay referencias de una recurrencia de entre el 2,4\% (Hardie y col 1983) y el 19\% (Dupre y col 1993). Hay otros autores que describen el uso de la transposición del obturador adicionándole un implante sintético, como una malla de polipropileno, en este caso tenemos una tasa de recurrencia del 12,5\% (Szabo 2007). La anatómica recoge en nuestro estudio tasas de recurrencia mucho más altas, en otro estudio en el que también se usaron ambas técnicas, describe una tasa de recidiva del $8 \%$ y dicho porcentaje se ciñe exclusivamente a la anatómica. Este hecho tal vez se deba a que con dicha técnica no se consigue, en algunos casos, cubrir el defecto ventral de la hernia, algo que sí se consigue con la transposición del obturador interno. Con los datos obtenidos no se recomienda la técnica anatómica para la resolución de la hernia perineal.

En cualquier caso, las tasas de recidiva encontradas en la transposición del obturador interno son muy bajas; está claro que requiere un aprendizaje previo para reducir las recidivas por inexperiencia del cirujano, pero debe considerarse una técnica fiable y segura para la herniorrafia perineal. Así mismo, no se considera necesario adicionar una malla de polipropileno a la técnica, ya que tanto nuestros resultados como los encontrados en la bibliografía no muestran una disminución significativa de las recidivas, además de que la colocación de la malla supone un coste extra, que no parece necesario, y prolonga el tiempo de cirugía.

Las técnicas complementarias suelen utilizarse para mejorar el resultado de la herniorrafia. En los casos en los que hay una hernia de gran tamaño que puede incorporar la vejiga en retroflexión o un gran falso divertículo rectal, la herniorrafia puede no ser suficiente para resolver las patologías derivadas de la hernia. La colopexia y la deferentopexia son técnicas usadas frecuentemente en el tratamiento de esas hernias perineales; la cistopexia no es una técnica de uso habitual, ya que con la herniorrafia y la deferentopexia es suficiente para mantener la vejiga en la cavidad abdominal. En nuestro estudio las tasas de recidiva son mayores, $22 \%$ frente a $13,32 \%$, combinando estas técnicas con la herniorrafia. Tal vez sea debido a que se utilizan en los casos más extremos y en estos casos el pronóstico es peor. Otros estudios describen el tratamiento de la hernia perineal combinando la herniorrafia y técnicas complementarias y obtienen tasas de recidiva inferiores y similares a la nuestra, 22\% (Maute y col 2001) y 10\% (Brissot y col 2004). 
En nuestra población se han obtenido 8,2\% de complicaciones, sin tener en cuenta las recidivas, corresponde a una tasa muy baja comparándola con lo que otros autores describen, 35\% (Hosgood 1995) y 26,7\% (Vnuk y col 2008), siendo un resultado satisfactorio. Las complicaciones que se han observado son similares a las descritas por los autores anteriores, incluyendo infección de la incisión, laxitud de la piel, seroma, y no resolución de la hernia.

En conclusión, la técnica de transposición del obturador interno ha sido la más fiable para la herniorrafia perineal y en las hernias perineales bilaterales hay mayor probabilidad de que exista retroflexión vesical.

\section{AGRADECIMIENTOS}

El autor agradece al Dr. Luis Javier Ezquerra Calvo, sin él nada habría sido posible, por su paciencia y su total dedicación a la enseñanza de la Cirugía Veterinaria, y en particular por alentarme a mejorar cada día. Agradezco a todos mis compañeros, el personal del Hospital Clínico Veterinario y a los alumnos que han aportado a este trabajo, quienes hacen el quehacer mucho más fácil.

\section{REFERENCIAS}

Bellenger R, RB Canfield. 2003. Perineal Hernia. In: Slatter D (ed). Textbook of Small Animal Surgery. Vol. 2. $3^{\text {rd }}$ ed. Saunders, Philadelphia, USA, Pp 487-498.

Brissot HN, GP Dupré, BM Bouvy. 2004. Use of laparotomy in a staged approach for resolution of bilateral or complicated perineal hernia in 41 dogs. Vet Surg 33, 412-421.

Burrows CF, CE Harvey. 1973. Perineal hernia in the dog. J Sm Anim Pract 14, 315-322.

Canfield RB. 1986. Anatomical aspects of perineal hernia in the dog. Doctoral Thesis. University of Sydney, Sydney, Australia.

Dupré GP, N Prat, B Bouvy. 1993. Perineal hernia in the dog: Evaluation of associated lesions and results in 60 dogs. Vet Surg 22, 250.

Fossum TW. 2007. Small Animal Surgery. ${ }^{\text {rd }}$ ed. Mosby Elsevier, St. Louis, USA, Pp 515-520.
Hardie EM, RJ Kolata, TD Early, CA Rawlings, CJ Gorgacz. 1983. Evaluation of internal obturator muscle transposition in treatment of perineal hernia in dogs. Vet Surg 12, 69-72.

Hayes HM Jr, GP Wilson, R E Tarone. 1978. The epidemiologic features of perineal hernia in 771 dogs. J Small Anim Hosp Assoc 14, 703-707.

Head LL, DA Fracis. 2002. Mineralized paraprostatic cyst as a potential contributing factor in the depelopment of perineal hernias in a dog. $J$ Am Vet Med Assoc 221, 533-535.

Hosgood G, C Hedlund, R Pechman, P Dean. 1995. Perineal herniorraphy: Perioperative data from 100 dogs. J Small Anim Pract 31, 331-342.

Mann FA. 1993. Perineal herniation. In: Bojrad MJ (ed). Disease mechanisms in small animal surgery. $2^{\text {nd }}$ ed. Lippincott Williams \& Wilkins, Philadelphia, USA, Pp 92-96.

Mattheison DT. 1989. Diagnosis and management of complications occurring after perineal herniorrhaphy in dogs. Comp Cont Ed Pract Vet 11, 797-822.

Maute AM, DA Koch, PM Montavon. 2001. Perineal hernia in dogs: colopexy, vasopexy and castration as elective therapies in $32 \mathrm{dogs}$. Schweiz Arch Tierheilkd 143, 360-367.

Pérez-Gutiérrez JF, JC Argüelles, M Iglesias-Núñez, KS Oliveira, M Sánchez de la Muela. 2011. Epidermal growth factor and active caspase- 3 expression in the levator ani muscle of dogs with and without perineal hernia. J Small Anim Pract 52, 365-370.

Pratummintra K, S Chuthatep, W Banlunara, M Kalpravidh. 2013. Perineal hernia repair using an autologous tunica vaginalis communis in nine intact male dogs. $J$ Vet Med Sci 75, 337-341.

Tobias KM. 2010. Manual of Small Animal Soft Tissue Surgery. WileyBlackwell, Iowa, USA, Pp 339-346.

Sontas BH, SÖ Apaydin, TSF Toydemir, G Kasikci, H Ekici. 2008. Perineal hernia because of retroflexion of the urinary bladder in a rottweiler bitch during pregnancy. J Small Anim Pract 49, 421-425.

Szabo S, B Wilkens, RM Radasch. 2007. Use of polipropilene mesh in addition to internal obturator transposition: A review of 59 cases (2000-2004). J Am Anim Hosp Assoc 43, 136-142.

Vnuk D, M Lipar, D Matičić, O Smolen, M Pećin, A Brkić. 2008. Comparison of standard perineal herniorrhaphy and transposition of the internal obturator muscle for perineal hernia repair in the dog. Vet Archiv 78, 197-207.

Weaver AD, JO Omamegbe. 1981. Surgical treatment of perineal hernia in the dog. J Small Anim Pract 22, 749-758.

White RAS, Herrtage ME. 1981. Bladder retroflexion in the dog. J Small Anim Pract 22, 735-746. 
